\title{
Sensing Characteristics of Hollow-Core Photonic Crystal Fibre Modal Interferometers
}

\author{
S. H. Aref ${ }^{\mathrm{a}, \mathrm{b}}$, R. Amezcua-Correa ${ }^{\mathrm{c}}$, J. P. Carvalho ${ }^{\mathrm{a}, \mathrm{d}}$, O Frazão ${ }^{\mathrm{a}, \mathrm{d}}$, P. Caldas ${ }^{\mathrm{a}, \mathrm{d}, \mathrm{e}}$, J. L. Santos ${ }^{\mathrm{a}, \mathrm{d}}$, F. M. Araújo ${ }^{\mathrm{a}}$, \\ H. Latifi ${ }^{b}$, F. Farahi ${ }^{\text {f }}$ L. A. Ferreira ${ }^{\text {a }}$ J. C. Knight ${ }^{\mathrm{c}}$ \\ ${ }^{a}$ INESC Porto, Rua do Campo Alegre, 687, 4169-007 Porto, Portugal \\ ${ }^{\mathrm{b}}$ Laser and Plasma Research Institute, Shahid Beheshti University, Evin, Tehran, Iran \\ ${ }^{c}$ Department of Physics, University of Bath, Claverton Down, Bath, BA2 7AY, UK \\ ${ }^{\mathrm{d}}$ Dept. de Física da Faculdade de Ciências da Universidade do Porto, Rua do Campo Alegre 687, 4169-007 Porto, Portugal. \\ ${ }^{e}$ Escola superior de Tecnologia e Gestão de Viana do Castelo, Av. do Atlântico, Apartado 574, 4900-348 Viana do Castelo, Portugal \\ ${ }^{\mathrm{f}}$ Department of Physics and Optical Science, University of North Carolina at Charlotte, Charlotte, NC 28223, USA
}

\begin{abstract}
In this work, sensitivity to strain, temperature and curvature of a sensor relying on modal interferometry in hollow-core photonic crystal fibre is studied. The sensing structure is simply a piece of hollow-core fibre connected in both ends to standard single mode fibre. An interference pattern that is associated to the interference of the light that propagates in the hollow core fundamental mode with light that propagates in other modes is observed. The phase of this interference pattern changes with the measurand interaction, which is the basis for considering this structure for sensing. The phase recovery is performed using a white light interferometric technique.
\end{abstract}

Keywords: Hollow-core photonic crystal fibre, fibre sensors, white light interferometry.

\section{Introduction}

The development of sensors based on hollow-core photonic crystal fibres (HC-PCF) has been a recent and active research topic in the context of fibre optic sensing. Particularly relevant is the application of these fibres for gas sensing in face of the large overlap of the optical field with the measurement volume [1], but other measurands have also been considered, for example bend and shape [2], strain and temperature [3], as well as hydrostatic pressure [4]. HC-PCF are made with hundreds of periodically spaced air holes in a silica matrix, typically arranged in a triangular lattice, using the photonic band gap concept to propagate light inside the core. HC-PCFs are multi-mode waveguides and other than the fundamental core mode, they can support higher order core modes, cladding modes and surface modes. However, the attenuation of the different types of modes varies a lot and after propagation along a long length of fibre only the fundamental core mode exists. The multi-mode operation of short lengths of HC-PCFs brings the possibility to build up modal interferometers with characteristics potentially interesting to perform optical fibre sensing.

In this work, a fibre optic modal interferometer based on HC-PCF was studied and its characteristics for sensing were investigated. The measurands addressed were strain, temperature and curvature. The readout of the interferometric phase was achieved combining white light addressing with pseudo-heterodyne signal processing.

\section{Sensing Principle}

The sensor was fabricated using a piece of HC-PCF directly spliced between two lengths of single mode fibre (SMF$28^{\mathrm{TM}}$ ) using a conventional fusion splice machine (Fujikura SM40). A 7-cell HC-PCF with a core diameter of $\approx$ $16 \mu \mathrm{m}$ was used. Figure 1 shows the SEM image of this fibre and its transmission characteristics in the light injection conditions in which the experiments were performed. The developed sensing head uses the effect of modal interference at the exit of the HC-PCF to achieve measurement functionality. Indeed, when light travels through the single mode fibre and is injected into the HC-PCF the fundamental core mode (FM) and other modes are excited. After propagation of these modes in the HC-PCF, they become recombined at the output single mode fibre. This simple configuration is similar to an all-fibre Mach-Zehnder modal interferometer with two coupling points in series. Therefore, when light is injected into the input single mode fibre, it is expected to observe interference fringes at the output single mode fibre. It should be noticed that the fringes are not related to the collapse of air channels of the HC-PCF, like tapering in the fusion splice point and/or broadening of fundamental mode in this region, as reported in the literature [7]. In order to confirm this, some experiments were carried out to check if the fringes still appear with butt coupling instead of fusion splicing. The results obtained showed that the formation of the interference fringes is independent of the coupling method. 

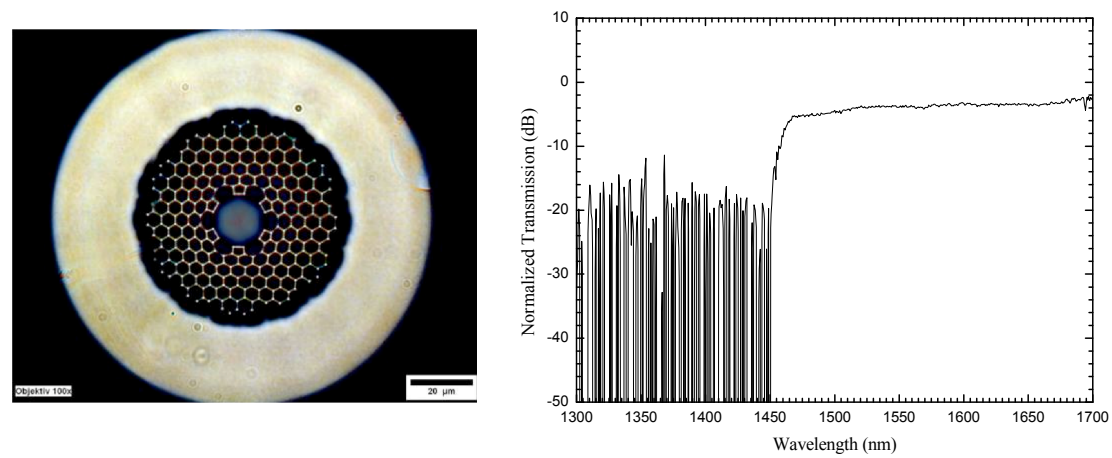

Figure 1. SEM image of a 7 cell HC-PCF (left) and normalized spectral transmission of $\sim 1 \mathrm{~m}$ of PCF fibre spliced to a SMF28 illuminating fibre (right; the oscillations at lower wavelengths are artifacts due to the normalization).

Figure 2 shows the registered channeled spectrum for a piece of HC-PCF with $27.8 \mathrm{~cm}$. It can be observed that besides the typical two-wave interferometric behavior, there is also fringe amplitude modulation, indicating the presence of other excited modes. The refractive index difference associated with the observed fringe periodicity in the figure can be calculated by means of the expression:

$$
n_{\text {eff }}^{F M}-n_{\text {eff }}^{\text {OtherMode }}=\frac{\lambda_{1} \lambda_{2}}{L\left(\lambda_{1}-\lambda_{2}\right)}
$$

In this relation $n_{\text {eff }}^{F M}$ and $n_{\text {eff }}^{\text {OtherMode }}$ are, respectively, the effective refractive index of the fundamental mode and of the higher order mode predominantly excited in the experiment performed. Also, $\lambda_{1}$ and $\lambda_{2}$ are the wavelengths corresponding to two adjacent intensity maxima and $L$ is the length of HC-PCF. It was found that the value of $\Delta n_{\text {eff }}=n_{\text {eff }}^{F M}-n_{\text {eff }}^{\text {OtherMode }}$ is around 0.016 , independently of $L$.

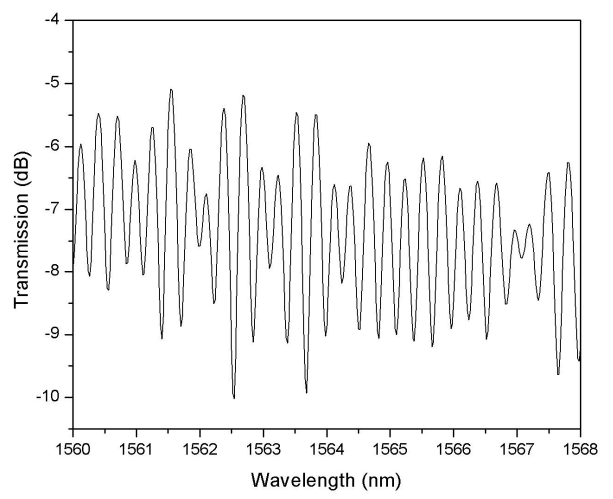

Figure 2. The channeled spectrum of the interferometric sensing head.

Keeping in mind that the core diameter of the illuminating fibre is smaller than that of the hollow core HC-PCF, it is possible that the extra modes are cladding modes. In any case, as shown below, it was found that their presence do not degrade noticeably the sensing characteristics of this fibre structure.

\section{Experimental Setup and Results}

The initial experimental set up is shown in Figure 3. An Erbium ASE source, operating at $1550 \mathrm{~nm}$,with a FWHM of 100 $\mathrm{nm}$ and output power of $2 \mathrm{~mW}$ was used to illuminate the concatenated fibres. By means of a conventional fusion splicer the HC-PCF was spliced in both ends to single mode fibres. All splicing process was done in manual mode and the optimum parameters were obtained after some attempts to achieve minimum fusion loss. This resulted in 0 bit and 400 ms for the power and arc time duration, respectively, and a typical splice loss of $1.5 \mathrm{~dB}$. The length of the hollow-core 
fibre was $\sim 28 \mathrm{~cm}$. In order to investigate the strain, temperature and curvature characteristics of the fibre sensor, two micro-positioners were used to fix the SMF fibres and apply strain and curvature. Figure 4 shows the strain and temperature wavelength responses of the sensing head obtained by monitoring the shift of the channeled spectrum under the measurand action. There are clear linear dependencies, with slopes $-0.92 \mathrm{pm} / \mu \varepsilon$ and $-7.1 \mathrm{pm} /{ }^{\circ} \mathrm{C}$. On the other hand, concerning curvature, no measurable sensitivity was noticed.

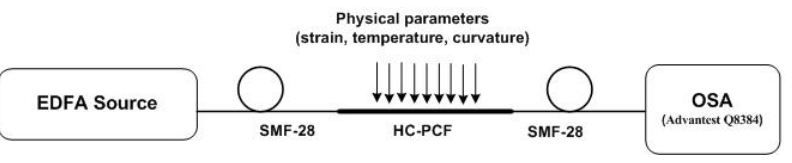

Figure 3. Experimental setup.
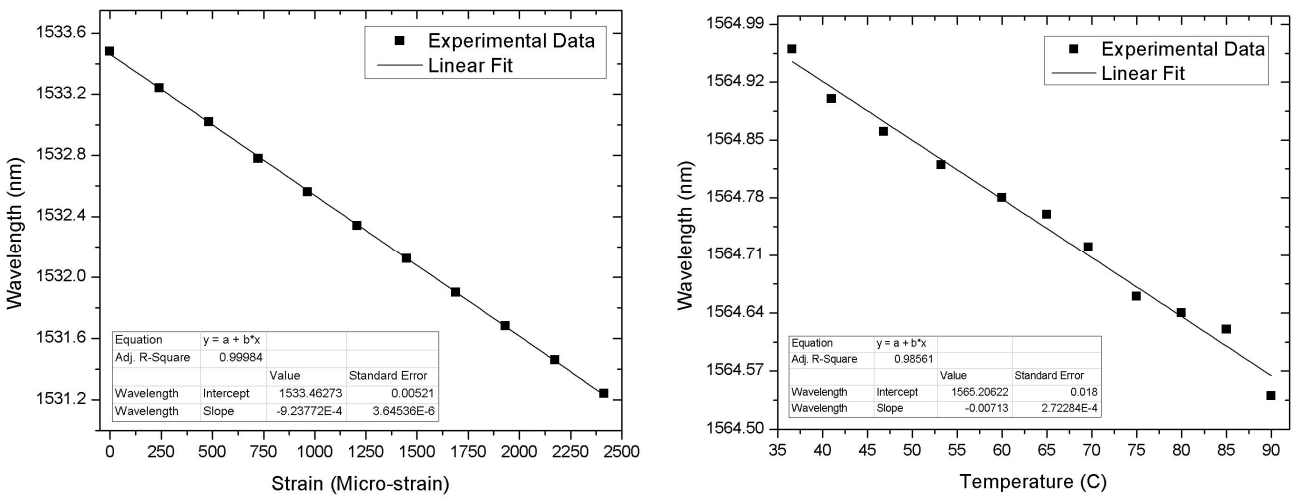

Figure 4. Wavelength responses of the sensing head for variations of applied strain and temperature.

To measure the phase changes in the fibre modal interferometer, a second interferometer was built to implement coherence reading [9]. Figure 5 shows the setup implemented. The second interferometer is a conventional fibre Michelson interferometer with an open air path in one of its arms, which is adjusted to match the optical path difference of the sensing interferometer. The fibre in the other arm of this interferometer is wrapped around a ring-shaped piezoelectric transducer that is modulated with an electrical sawtooth waveform whose amplitude is adjusted to obtain a signal at the photodetection suitable for pseudo-heterodyne processing. After an adequate electronic filtering, this signal has the form of an electric carrier $(90 \mathrm{~Hz})$ with a phase that mirrors the optical phase of the tandem interferometric system. This pseudo-heterodyne processing technique is known to provide sensitive interferometric phase reading [9].

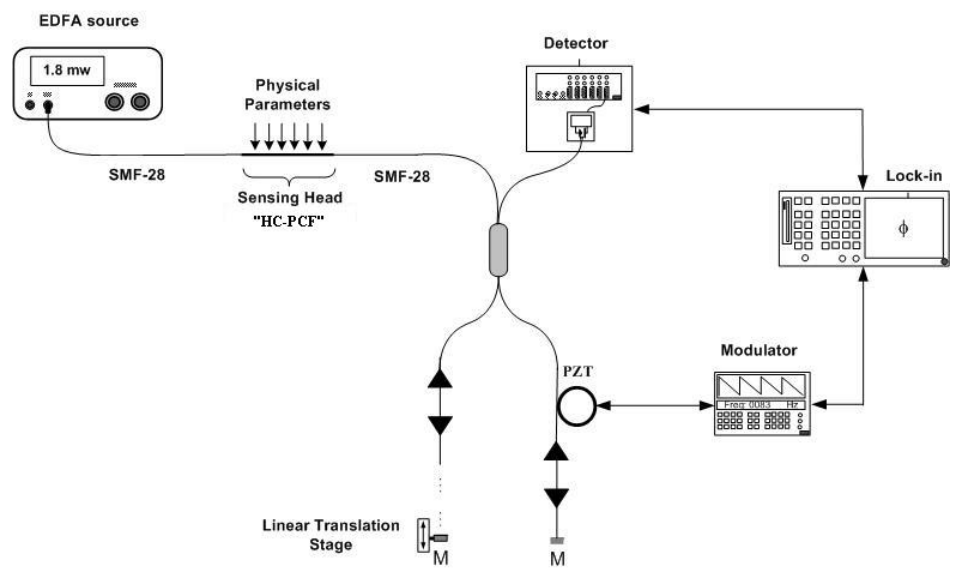

Figure 5. Scheme of the experimental setup for phase reading with white light interferometry.

Figure 6 shows the phase changes associated with strain and temperature variations applied to the sensing head, now with $\mathrm{L} \approx 40 \mathrm{~cm}$. Following the behavior represented in Figure 5, the sensitivity is constant in the measurement range considered, with values of $0.76^{\circ} / \mu \varepsilon$ and $8.1^{\circ} /{ }^{\circ} \mathrm{C}$. To evaluate the measurand resolutions achievable with this sensing head 
structure, the sensor output was registered during a measurand step change. From the phase rms fluctuations during the periods of constant measurand values and the phase step change induced by a corresponding measurand step, resolutions of $\pm 1.4 \mu \varepsilon$ and $\pm 0.2^{\circ} \mathrm{C}$ were obtained for strain and temperature, respectively.
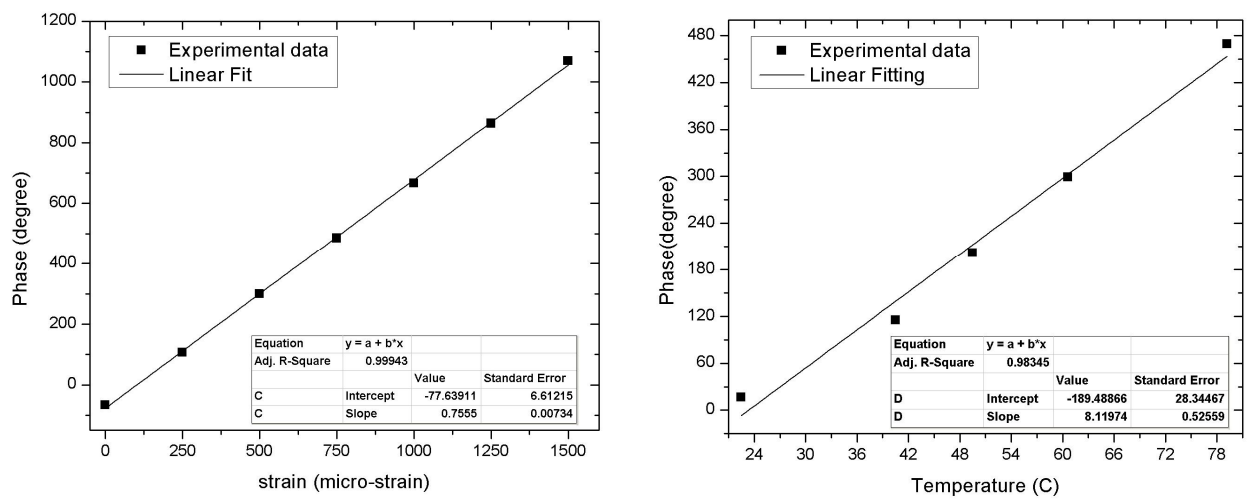

Figure 6 . Phase changes induced by strain and temperature variations applied to the sensing head.

\section{Conclusions}

A sensing head based on hollow-core photonic crystal fibre for measurement of strain and temperature has been presented. The sensor consists of a piece of 7-cell HC-PCF connected to SMF-28 in both ends. The interference occurs between the fundamental mode and higher order modes inside the HC-PCF. A white light interferometric technique for coherent phase reading was used. Resolutions of $\pm 1.4 \mu \varepsilon$ and $\pm 0.2^{\circ} \mathrm{C}$ were obtained for strain and temperature, respectively. It was also found that the fibre structure is not sensitive to curvature.

\section{Acknowledgments}

The authors acknowledge the R\&D environment provided by the FP6 Project NextGenPCF (IST - 34918: Next Generation Photonic Crystal Fibres).

\section{References:}

[1] R. Thapa, K. Knabe, K. L. Corwin, B. R. Washburn, "Arc fusion splicing of hollow-core photonic bandgap fibres for gas-filled fibre cells", Opt. Express 14(21), 9576-9583 (2006).

[2] W. N. MacPherson, M. J. Gander, R. McBride, J. D. C. Jones, P. M. Blanchard, J. G. Burnett, A. H. Greenaway, B. Mangan, T. A. Birks, J. C. Knight, P. St. J. Russell, "Remotely addressed optical fibre curvature sensor using multicore photonic crystal fibre", Opt. Comm., 193(1-6), 97-104 (2001).

[3] Chun-Liu Zhao, Limin Xiao, Jian Ju, M. S. Demokan, Wei Jin, "Strain and Temperature Characteristics of a LongPeriod Grating Written in a Photonic Crystal Fibre and Its Application as a Temperature-Insensitive Strain Sensor", J. Lightwave Tech., 26, 220-227 (2008).

[4] William N. MacPherson, Euan J. Rigg, Julian D. C. Jones, V. V. Ravi Kanth Kumar, Jonathan C. Knight, Philip St. J. Russell, "Finite-Element Analysis and Experimental Results for a Microstructured Fibre With Enhanced Hydrostatic Pressure Sensitivity", J. Lightwave Tech. 23, 1227-31 (2005).

[5] M. N. Petrovich, F. Poletti, A. van Brakel, D. J. Richardson, "Robustly single mode hollow core photonic bandgap fibre", Opt. Express 16, 4337-4346 (2008).

[6] R. F. Cregan, B. J. Managan, J. C. Knight, T. A. Birks, P. St. J. Russell, P. J. Roberts, D. C. Allen, "Single-mode photonic band gap guidance of light in air", Science 285, 1537-9 (1999).

[7] Joel Villatoro, Vladimir P. Minkovich, Valerio Pruneri, Gonçal Badenes, "Simple all-microstructured-optical-fibre interferometer built via fusion splicing", Opt Express 15, 1491-1496 (2007).

[8] Y.J. Rao, D.A. Jackson, "Review article: Recent progress in fibre-optic low-coherence interferometry", Meas. Sci. Tech. 7, 981-999 (1996).

[9] P. Caldas, P. A. S. Jorge, F. M. Araujo, L. A. Ferreira, M. B. Marques, G. Rego, J. L. Santos, "Fibre modal Michelson interferometers with coherence addressing and heterodyne interrogation", Opt. Eng. 47(4), 44401 (2008). 